\title{
Real-time Vision-Based Microassembly of 3D MEMS
}

\author{
Brahim Tamadazte, Thomas Arnould, Sounkalo Dembélé, Nadine Le Fort-Piat and Eric Marchand
}

\begin{abstract}
Robotic microassembly is a promising way to fabricate micrometric components based three dimensions (3D) compound products where the materials or the technologies are incompatible: structures, devices, Micro Electro Mechanical Systems (MEMS), Micro Opto Electro Mechanical Systems (MOEMS),... To date, solutions proposed in the literature are based on 2D visual control because of the lack of accurate and robust 3D measures from the work scene. In this paper the relevance of the real-time $3 \mathrm{D}$ visual tracking and control is demonstrated. The 3D poses of the MEMS is supplied by a model-based tracking algorithm in real-time. It is accurate and robust enough to enable a precise regulation toward zero of a 3D error using a visual servoing approach. The assembly of $400 \mu \mathrm{m} \times \mathbf{4 0 0} \mu \mathrm{m} \times 100 \mu \mathrm{m}$ parts by their $100 \mu \mathrm{m} \times 100 \mu \mathrm{m}$ $\times 100 \mu \mathrm{m}$ notches with a mechanical play of $3 \mu \mathrm{m}$ is achieved with a rate of 41 seconds per assembly. The control accuracy reaches $0.3 \mu \mathrm{m}$ in position and $0.2^{\circ}$ in orientation.
\end{abstract}

\section{INTRODUCTION}

In the past decade the need of automated microassembly systems i.e. (systems that fit together micrometric devices like: MEMS, MOEMS or BioMEMS (Biomedical Micro Electro Mechanical Systems) become more and more necessary because of the growing interest in such products. Consequently, an active and intensive research area appeared. Pursuit of the minimization of the devices, fabrication of new 3D complex products and the assembly of hybrid microsystems constituted of incompatible materials and produced using various technologies. Hence, many microassembly workcell have been developed and presented in the literature by different laboratories. Dechev et al [5], Nelson and his colleagues presented a 6 degrees of freedom (dof) microassembly workcell in [19] and Popa et al [4] developed a fully reconfigurable 19 dof microassembly station. The major work in the automatic microassembly of microsystems have a common point that is the use of sensory feedback control. This feedback control consider vision sensors (via a microscope) [17], [6], force sensors integrated with the gripper, or both [16], [20], [2], [19]. A perfect assembly station would consist on of flexible, versatile, modular, precise and repeatable system. Precisions should be below the micrometer and the MEMS assembly cadence would be about one complex microsystem product/minute.

This paper focuses in the assembly of 3D solid structures by a high precision 5 dof microassembly workcell. It includes a 3 dof positioning platform and a 2 micromanipulator that

Brahim Tamadazte, Thomas Arnould, Sounkalo Dembélé, Nadine Le Fort-Piat are from FEMTO-ST Institute-AS2M/UMR CNRS 6174 /UFC/ ENSMM / UTBM, 24 rue Alain Savary, 25000 Besançon, France, brahim.tamadazte@ens 2 m. fr

Eric Marchand is from INRIA Rennes-Bretagne Atlantique, IRISA, Lagadic, France, ericamarchandeirisa.fr

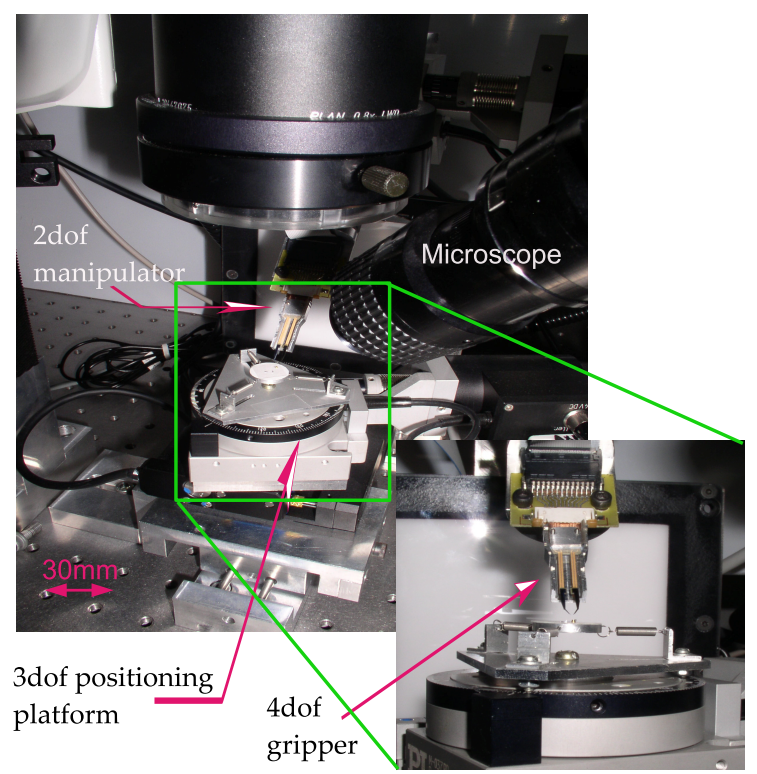

Fig. 1. The five degrees of freedom microassembly workcell

supports the gripping system, one mono-view vision sensor system and the silicon parts to assemble. $3 \mathrm{D}$ visual control is used to fit together a silicon micropart $\mathbf{A}$ on silicon micropart B whose size are [400 $\mu \mathrm{m} \times 400 \mu \mathrm{m} \times 100 \mu \mathrm{m}$ ], Fig. 7 . These microparts are tracked by means of an algorithm based on their CAD model. It is proved that high speed and high precision assembly of $3 \mathrm{D}$ microstructures is possible. The task is performed in real-time, ie at the frequency of acquisition of the videomicroscope ( 7.5 frames by second). The cycle time is about 41 seconds, the positioning error is submicrometric and the orientation error is below one degree.

This paper is structured as followed: In section [II], the microassembly workcell, based on high precision linear, angular motions, imaging system, gripping system, clean box,... Section [III] describes the 3D model-based tracking algorithm used to track in real-time the microparts in the images of the videomicroscope. Section [IV] presents the $3 \mathrm{D}$ visual control that consists in regulation to zero of a $3 \mathrm{D}$ pose error. Section [V] demonstrates the relevance of the concepts and methodology proposed in this paper in terms of assembly precision, speed and robustness.

\section{THE Microassembly WORKCELL}

\section{A. The 5 dof Robotic System}

The robotic workstation used for the microassembly process is illustrated in Fig. 2 in a kinematic point of view. The 
robotic structure is separated into two independent systems: the first system is a $3 \operatorname{dof}(x y \theta)$ positioning platform, and the second system is a micromanipulator with 2 dof $(z \phi)$. The translation motions $(x, y, z)$ are characterized by a resolution $r=0.007 \mu \mathrm{m}$, an increment $i=0.05 \mu \mathrm{m}$, a velocity $v_{t}=$ $1.5 \mathrm{~mm} / \mathrm{s}$, a stroke of $s_{t}=25 \mathrm{~mm}$. The specifications of the angular motions are: $r=26 \mu \mathrm{rad}, i=26 \mu \mathrm{rad}$ and speed $v_{r}=$ $45 \mathrm{deg} / \mathrm{s}$. The former system is equipped with a compliant table (the table is supported by three springs) that enables the positioning in the horizontal plane. The latter system supports the gripper and enables the vertical positioning and spatial orientation of the parts.

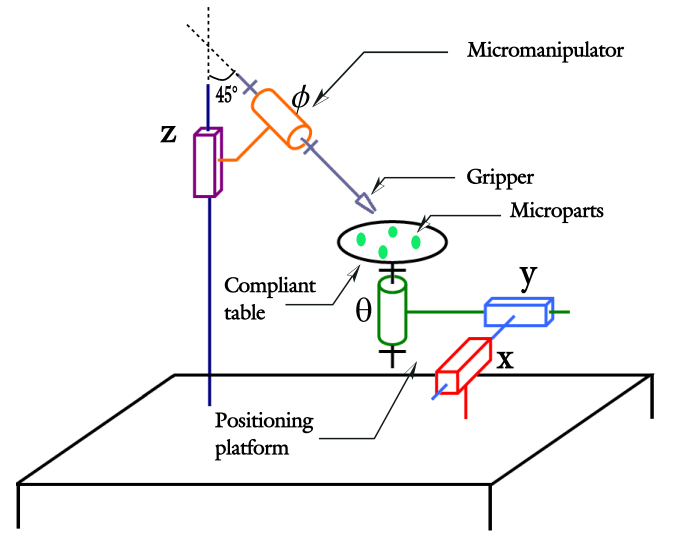

Fig. 2. The mechanical structure of the microassembly workcell

\section{B. Gripping System}

A Microprehensile Microrobot On Chip (MMOC) developed in the Automatic Control and Micro-Mechatronic Systems Department (AS2M) of Femto-ST Institute is used for the handling of parts (Fig. 1). It is a two-fingers gripping system with four dof (two dof by finger) which enables openand-close motions as well as up-and-down motions. Every finger is a piezoelectric bimorph with two silicon layers (12 and $400 \mu \mathrm{m})$ separated by an oxyde layer $(1 \mu \mathrm{m})$.

Modularity is an important design criterion during development, and the MMOC microgripper has been designed to use different end-effectors (finger tips). Then it can grab a high variety of objects according to the kind of endeffectors: planar silicon parts, balls, gears, optical fibers,... The specimen used in the current experiments are endowed with nickel end-effectors, and its corresponding features and performances are summarized in Table I.

\section{The Imaging System}

Only one CCD camera (ALLIED AVT Marlin F-033B) with a resolution of $[1280 \times 960]$ pixels is used. It is mounted at $43^{\circ}$ from the vertical axis and is equipped with a microscope. It provides a field of view of $1.216 \mathrm{~mm}$ $\times 0.912 \mathrm{~mm}$, a resolution about $0.95 \mu \mathrm{m}$ and a working distance of $80 \mathrm{~mm}$. The lens-camera system is mounted on two manual translation stages in order to adjust its position. The acquisition frequency can be adjusted, in our experiment, it has been limited to $F=7.5$ images $/ \mathrm{s}$.

\begin{tabular}{|l|c|}
\hline \multicolumn{2}{|c|}{ Typical strokes } \\
\hline $\begin{array}{l}\text { open-close } \\
\text { up-and-down }\end{array}$ & $320 \mu \mathrm{m}$ \\
\hline \multicolumn{2}{|c|}{ Blocking forces } \\
\hline open-close & $55 \mathrm{mN}$ \\
up-and-down & $10 \mathrm{mN}$ \\
\hline \multicolumn{2}{|c|}{ Other characteristics } \\
\hline High resolution & $\sim 10 \mathrm{~nm}$ \\
Speed & $\prec 10 \mathrm{~ms}$ \\
\hline
\end{tabular}

TABLE I

THE MOCC MICROGRIPPER FEATURES

\section{The Microparts}

In this paper we target the assembly by insertion in each other of two parts of dimensions $400 \mu \mathrm{m} \times 400 \mu \mathrm{m} \times 100$ $\mu \mathrm{m}$. A notch of $100 \mu \mathrm{m} \times 100 \mu \mathrm{m} \times 100 \mu \mathrm{m}$ is engraved in every side of each part (Fig. 3). Let $A$ and $B$ be the microparts. The objective is to automatically insert a notch of $A$ into a notch of $B$. The mechanical play is about $3 \mu \mathrm{m}$ enabling a solid assemblage without any fixture.

That problem can be broken down into three basic tasks that should be performed sequentially: the displacement of the part $A$ (Task\#1), the displacement of the part $B$ (Task\#2) and its insertion into $A$ (Task\#3).

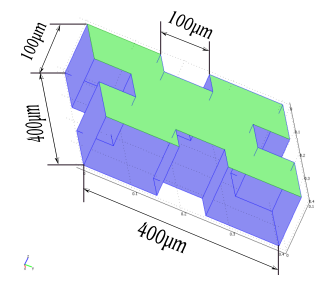

Fig. 3. An example of silicon micropart used for the assembly

\section{Robust MODEL-BASED TRACKING Algorithm}

To track the microscale object we considered a 3D modelbased tracker that allows the computation of the object pose. It relies Full-scale non-linear optimization techniques [9], [11], [7], [3] which consist of minimizing the error between the observation and the forward-projection of the model. In this case, minimization is handled using numerical iterative algorithms such as Newton-Raphson or LevenbergMarquardt. Let us note that such tracker have also been consider in the case of microsystems in [21].

The goal of tracker is to compute the position ${ }^{c} \mathbf{M}_{o}$ of the object in the camera frame ${ }^{1}$. In this paper, the pose computation is formulated in terms of a full scale non-linear optimization: Virtual Visual Servoing (VVS). In this way the

\footnotetext{
${ }^{1}$ Let us define the rigid transformation between a frame $\mathscr{R}_{a}$ and a frame $\mathscr{R}_{b}$ by an homogeneous matrix ${ }^{a} \mathbf{M}_{b}$ defined by:

$$
{ }^{a} \mathbf{M}_{b}=\left[\begin{array}{cc}
{ }^{a} \mathbf{R}_{b} & { }^{a} \mathbf{t}_{b} \\
0 & 1
\end{array}\right],
$$

where ${ }^{a} \mathbf{R}_{b}$ is the rotation matrix and ${ }^{a} \mathbf{t}_{b}$ the translation vector. It is also possible to note the pose by the vector ${ }^{a} \mathbf{r}_{b}=\left({ }^{a} \mathbf{t}_{b}, \theta \mathbf{u}\right)$ where $\theta \mathbf{u}$ is the axes and the angle of the rotation.
} 
pose computation problem is considered as similar to $2 \mathrm{D}$ visual servoing as proposed in [3]. Assuming that the low level data extracted from the image are likely to be corrupted, we use a statistically robust camera pose estimation process (based on the widely accepted statistical techniques of robust M-estimation [10]). This M-estimation is directly introduced in the control law to address [3]. This framework is used to create an image feature based system which is capable of treating complex scenes in real-time.

More precisely we minimizer the distances between contour point extracted from the image and the projection of the 3D lines of the CAD model of the object (see Fig. 3). let us denote $\mathbf{p}_{i}, i=1 . . k$ these points and $\mathbf{l}_{i}(\mathbf{r})$ the projection of the corresponding line for the pose $\mathbf{r}$

$$
\widehat{{ }^{c} \widehat{\mathbf{M}}_{o}}=\arg \min _{{ }^{2} \mathbf{R}_{o},{ }^{c} t_{o}} \sum_{i=1}^{k} \rho\left(d_{\perp}\left(\mathbf{p}_{i}, \mathbf{l}_{i}\left({ }^{c} \mathbf{r}_{o}\right)\right)\right)
$$

where $\rho($.$) is the robust function that allows to handle$ corrupted data. The distance $d_{\perp}($.$) is represented on Fig. 4$

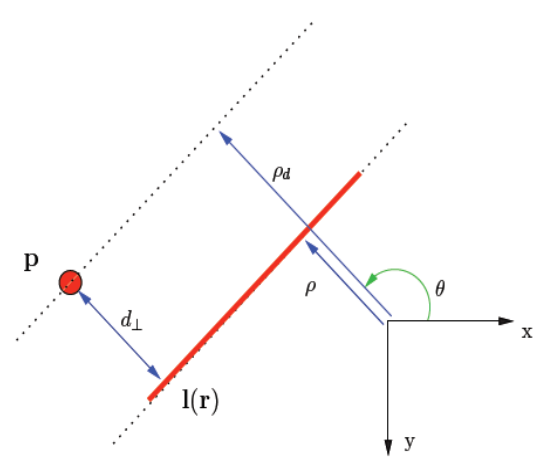

Fig. 4. Distance of a point to a line

Since a Gauss-Newton approach is considered to minimize equation (2) a Jacobian has to be defined and is given in [3].

\section{Modelling of the Assembly Process}

Although visual servoing has been widely considered in microassembly [21], [8], [15], [2], [17], most of system consider image-based visual servoing approach rather than position-based visual servoing (see [1] for a comprehensive description of IBVS and PBVS). At the macroscale, the positioning of a camera supported by a robot with respect to a target using 3D visual servo is widely considered [18], [14]. In that case the vision sensor is considered as a $3 \mathrm{D}$ sensor.

Until now, few publication [21] has investigated the assembly of microparts by implementing 3D visual control of the microscale.

The main advantage is that the task is describe as a $3 \mathrm{D}$ error (between the position of the two microparts to be manipulated) to be regulated to zero. The use of a 3D tracker that provides microparts 3D localization, which can be seen as a drawback, in fact provides a more robust to track the object in the image sequence.

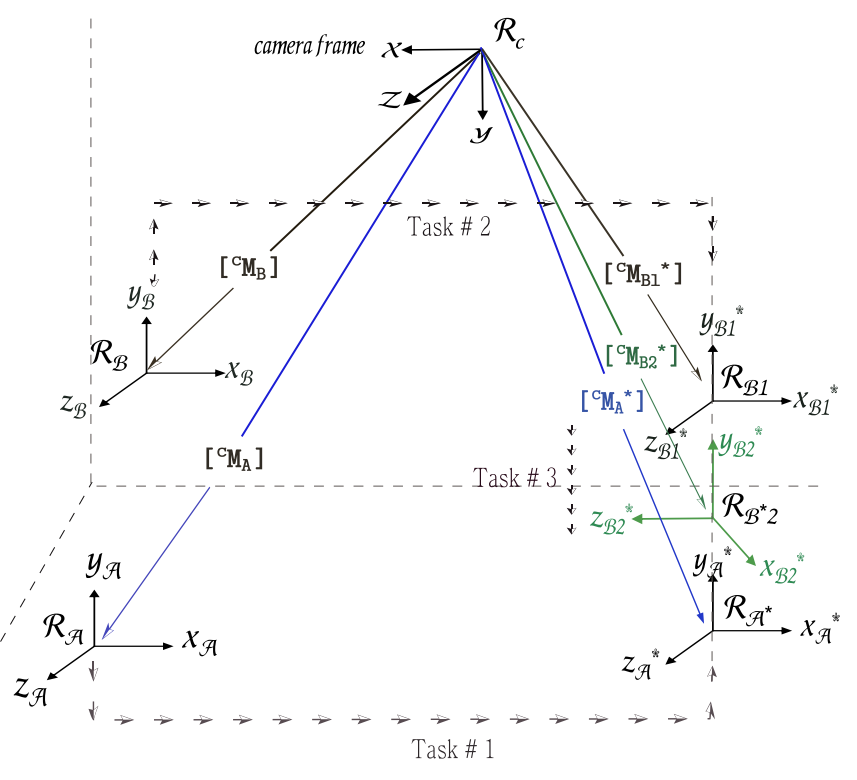

Fig. 5. Methodology of the insertion of the micropart $A$ into the micropart $B$

\section{A. Notations}

Let $\mathscr{R}_{c}, \mathscr{R}_{A}, \mathscr{R}_{A^{*}}, \mathscr{R}_{B}, \mathscr{R}_{B_{2}^{*}}$, be respectively the frame attached to the camera (i.e. the video microscope), the current and final frames of the part $A$, current and final frames of the part $B$. Moreover, an insertion frame $\left(\mathscr{R}_{B_{1}}\right)$ for $B$ is required where the part process through before switching to the insertion stages.

The tracking algorithm is expected to supply each sample time the following information:

- ${ }^{c} \mathbf{M}_{A}$ the homogeneous transformation between the camera frame and the current position of $A$,

- ${ }^{c} \mathbf{M}_{B}$ the homogeneous transformation between the camera frame and the current frame of $B$.

Let

- ${ }^{c} \mathbf{M}_{A^{*}}$ be the homogeneous transformation between the camera frame and the desired frame of $A$,

- ${ }^{c} \mathbf{M}_{B_{1}^{*}}$ be the homogeneous transformation between the camera frame and the insertion frame of $B$,

- ${ }^{c} \mathbf{M}_{B_{2}^{*}}$ be the homogeneous transformation between the camera frame and the desired frame of $B$.

\section{B. Control Law}

Let us decompose the insertion task in three different tasks:

1) task 1: displacement of the micropart A to a given position (defined by $\mathscr{R}_{A^{*}}$ ); To achieve this task a control law has to regulate to zero an error defined from:

$$
{ }^{A} \mathbf{M}_{A^{*}}={ }^{c} \mathbf{M}_{A}^{-1 c} \mathbf{M}_{A^{*}},
$$

2) task 2: displacement of the second micropart B to an intermediate position (defined by $\mathscr{R}_{B_{1}^{*}}$ ). To achieve this 
task a control law has to regulate to zero an error defined from:

$$
{ }^{B} \mathbf{M}_{B_{1}^{*}}={ }^{c} \mathbf{M}_{B}^{-1}{ }^{c} \mathbf{M}_{B_{1}^{*}}
$$

3) task 3: insertion of micropart B in micropart A (defined by $\mathscr{R}_{B_{2}^{*}}$ ). To achieve this task a control law has to regulate to zero an error defined from:

$$
{ }^{B} \mathbf{M}_{B_{2}^{*}}={ }^{c} \mathbf{M}_{B}^{-1}{ }^{c} \mathbf{M}_{B_{2}^{*}}
$$

Switching between task 2 and 3 may be done when the error defined from ${ }^{B} \mathbf{M}_{B_{1}^{*}}$ is small enough.

As in most of visual servoing application, a key problem is the definition of the desired position of the object wrt the camera. In our case we have to determine ${ }^{c} \mathbf{M}_{A^{*}},{ }^{c} \mathbf{M}_{B_{1}^{*}}$, ${ }^{c} \mathbf{M}_{B_{2}^{*}}$. A learning approach has been considered in the current experiments, the process is performed by teleoperation and the corresponding poses are recorded. The definition of $\mathscr{R}_{B_{1}}$ with accuracy is important for the success of the insertion (Fig. 5) since that operation requires caution and accuracy. A solution using directly the CAD models of the part will be investigated in a near.

Let us define $\mathscr{R}_{\mathscr{F}}$ as the base frame of workcell. To regulate to zero the error defined from ${ }^{A} \mathbf{M}_{A^{*}}$ a solution is to choose as visual feature $\mathbf{s}=\left({ }^{\mathscr{F}} \mathbf{t}_{A}, \theta \mathbf{u}\right)$ where $\theta \mathbf{u}$ defines the angle and the axes of the rotation that the object has to realize (defined from ${ }^{A} \mathbf{R}_{A^{*}}$ ). In that case $\mathbf{s}^{*}=\left({ }^{\mathscr{F}} \mathbf{t}_{A^{*}}, \mathbf{0}\right)$.

${ }^{\mathscr{F}} \mathbf{M}_{A}$ can be computed as

$$
{ }^{\mathscr{F}} \mathbf{M}_{A}={ }^{\mathscr{F}} \mathbf{M}_{c}{ }^{c} \mathbf{M}_{A}
$$

where ${ }^{\mathscr{F}} \mathbf{M}_{c}$ is the position of the camera in the reference frame of the workcell (which is a known constant) and ${ }^{c} \mathbf{M}_{A}$ is given by the tracking algorithm presented in the previous section.

The equation that links the variation $\dot{\mathbf{s}}$ of the visual feature $\mathbf{s}$ to the robot velocity in the robot reference frame $(v, \omega)_{\mathscr{F}}^{\top}$ are given by [13]:

$$
\left(\begin{array}{c}
\mathscr{F} \dot{\mathbf{t}}_{A} \\
\dot{\theta \mathbf{u}}
\end{array}\right)=\left(\begin{array}{cc}
\mathbf{I}_{3 \times 3} & \mathbf{0}_{3 \times 3} \\
\mathbf{0}_{3 \times 3} & \mathbf{J}_{\omega}
\end{array}\right)\left(\begin{array}{c}
v \\
\omega
\end{array}\right)_{\mathscr{F}}
$$

where

$$
\mathbf{J}_{\omega}=\mathbf{L}_{\omega}{ }^{c} \mathbf{R}_{\mathscr{F}}
$$

where $\mathbf{L}_{\omega}$ is such that $\mathbf{L}_{\omega}^{-1} \theta \mathbf{u}=\theta \mathbf{u}$ [12].

Using a 6 dof robot, we would obtain the following control law:

$$
\begin{aligned}
\left(\begin{array}{c}
v \\
\omega
\end{array}\right)_{\mathscr{F}} & =-\lambda\left(\begin{array}{cc}
\mathbf{I}_{3 \times 3} & \mathbf{0}_{3 \times 3} \\
\mathbf{0}_{3 \times 3} & \mathbf{J}_{\omega}^{-1}
\end{array}\right)\left(\mathbf{s}-\mathbf{s}^{*}\right) \\
& =-\lambda\left(\begin{array}{c}
\mathscr{F} \mathbf{t}_{A}-{ }^{\mathscr{F}} \mathbf{t}_{A^{*}} \\
\mathscr{F} \mathbf{R}_{A} \theta \mathbf{u}
\end{array}\right)
\end{aligned}
$$

Considering task 1 , since only the $x, y$ and $\theta$ dof are considered, the controlled law is given by

$$
\left(\begin{array}{c}
\dot{x} \\
\dot{y} \\
\dot{\theta}
\end{array}\right)_{\mathscr{F}}=-\lambda_{1}\left(\begin{array}{c}
t_{x}-t_{x}^{*} \\
t_{y}-t_{y}^{*} \\
\mathscr{F} \mathbf{R}_{A} \theta u_{\theta}
\end{array}\right)
$$

where $\left(t_{x}, t_{y}, t_{z}\right)={ }^{\mathscr{F}} \mathbf{t}_{A}$ and $\left(t_{x}^{*}, t_{y}^{*}, t_{z}^{*}\right)={ }^{\mathscr{F}} \mathbf{t}_{A^{*}}$. Similarly, for the second and third task, since only the $z$ and $\phi$ dof are considered, the controlled law is given by

$$
\left(\begin{array}{c}
\dot{z} \\
\dot{\phi}
\end{array}\right)_{\mathscr{F}}=-\lambda_{2}\left(\begin{array}{c}
t_{z}-t_{z}^{*} \\
\mathscr{F} \mathbf{R}_{A} \theta u_{\phi}
\end{array}\right)
$$

The system switch automatically between task1, 2 and 3 when the norm of the error $\|\mathbf{e}\|$ is below a predefined threshold.

To improve the convergence rate, we have implemented an adaptive gain (the gain increases when the error decreases):

$$
\lambda_{a d p}=\left(\lambda_{\max }-\lambda_{\min }\right) \exp ^{-\kappa\|\mathbf{e}\|}+\lambda_{\text {min }}
$$

where $\lambda_{\max }$ and $\lambda_{\min }$ are the maximum and minimum values of $\lambda_{\text {adp }}$ respectively and $\kappa$ is a constant.

\section{RESUlTS}

\section{A. Qualitative Evaluation}

Figure 6 displays some images acquired by the camera during the positioning, centering, orientation and insertion tasks. The images 1 to 5 show the positioning of the part A with respect of the final pose (task\#1). The shots 6 and 7 show the positioning of the part $\mathbf{B}$ with respect to the insertion pose (task\#2), where as the shot 8 shows the end of the insertion (task\#3). The CAD model of the parts are reprojected in the images in order to indicate the recognition of the parts. These results indicate the relevance of the CAD model based tracking at the microscale. For the record the part size are [400 $\mu \mathrm{m} \times 400 \mu \mathrm{m} \times 100 \mu \mathrm{m}]$.

Figure 7 shows some Scanning electron microscope (SEM) images of the final assembly. The high quality of the result expresses the relevance of the control approach: insertion with a mechanical play of $3 \mu \mathrm{m}$ gives a solid structure that avoids the use of any fixing.

\section{B. Accuracy and Robustness}

Figure 8 shows the evaluation of the errors according to the number of iterations for on example of assembly. From left to right and up to down the figure represents respectively:

- The error of positioning along $x$ axis of the part $\mathbf{A}$ (task\#1) (the final value is $e_{x}=3.52 \mu \mathrm{m}$ ),

- The error of positioning along $y$ axis of the part $\mathbf{A}$ (task\#1) (the final value is $e_{y}=0.29 \mu \mathrm{m}$ ),

- The error of orientation in the horizontal plane of the part A (task\#1) (the final value is $e_{\theta}=0.17^{\circ}$,

- The error of positioning along $z$ axis of the part $\mathbf{B}$ (task\#2) (the final value is $e_{z}=2.28 \mu \mathrm{m}$ ),

- The error of positioning along $z$ axis of the part $\mathbf{B}$ (task\#3),

- The error of the orientation in the space of the part B (task\#3) (the final value is $e_{\phi}=0.80^{\circ}$.

These values are obtained by the encoders of the different angular and linear motions. The low level of these errors enabled the insertion despite the mechanical play of $3 \mu \mathrm{m}$.

The robustness of the approach presented in this paper can be underlined on several points: multiple temporary and partial occlusions (by the gripper or by other microparts) 


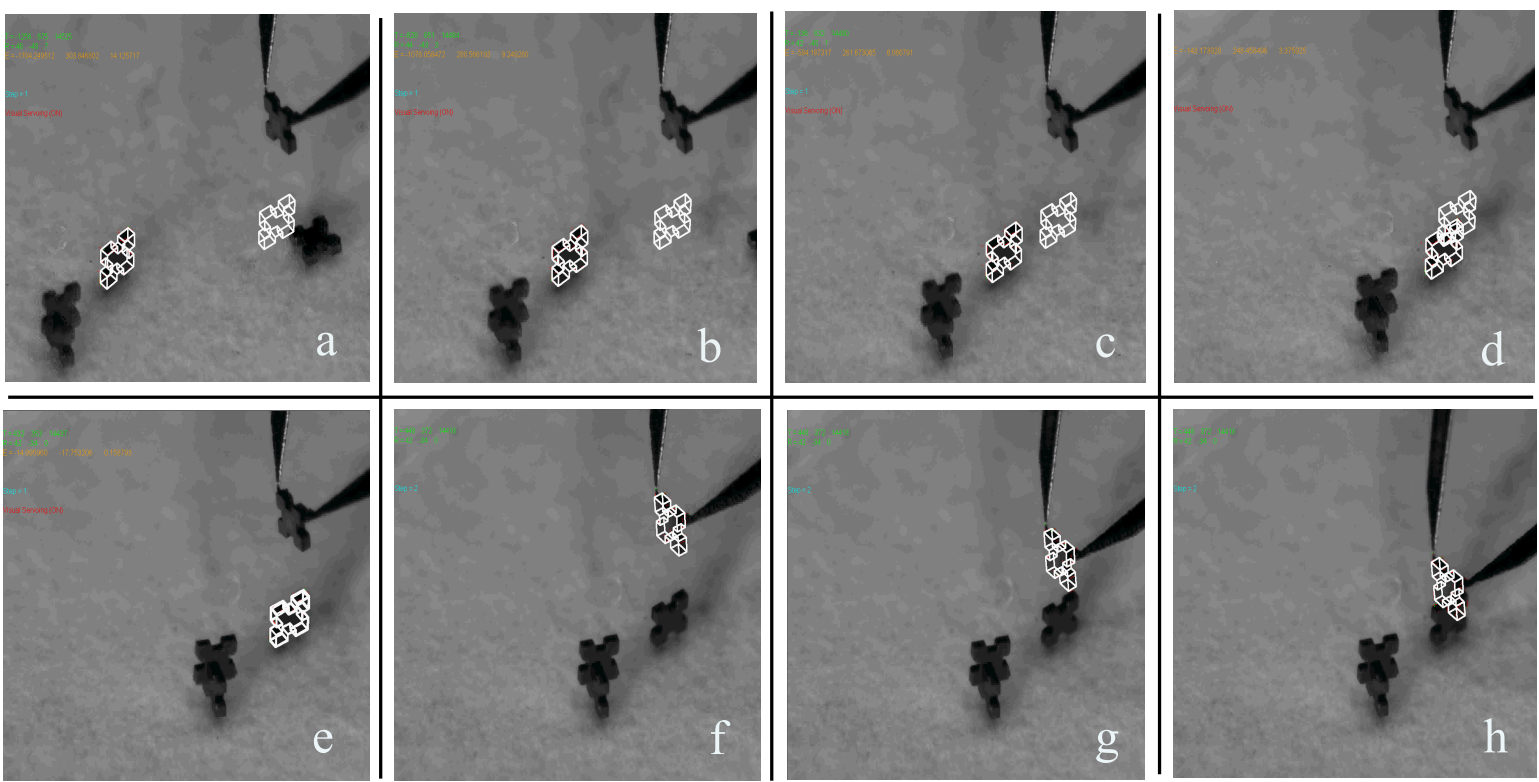

Fig. 6. Successful assembly of two parts : the image (a) represents the tracker initialization, the image (e) shows the end of the positioning of the part A, the image (f) shows the beginning pose of the part B, the image (h) shows the end of the assembly

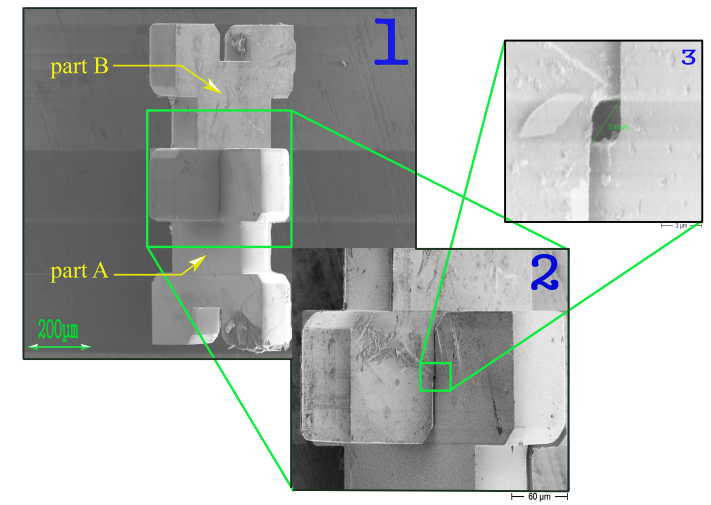

Fig. 7. (1) a successful example of two parts assembled and viewed on the SEM, (2) represents the zooming in on the part notch and (3) displays the high precision of the approach proposed above

of the microparts, happened during the manipulation. That did not prevent the algorithm to track the parts. In addition, because the weakness of the depth of field of the optical microscope can not allowed to view the microparts completely sharp. The tracking method based on the virtual visual servoing combined with a technique of robust Mestimation introduced, directly, on the control law permit to track precisely even with a blurred part of the component tracked.

\section{Assembly Cycle Time}

The mean cycle time for the single successful assembly by a human operator is about 10 minutes if that person has approximately one month of training. With a such time, it is almost impossible to make the product beneficial. The major part of that cycle time is the insertion which requires caution,
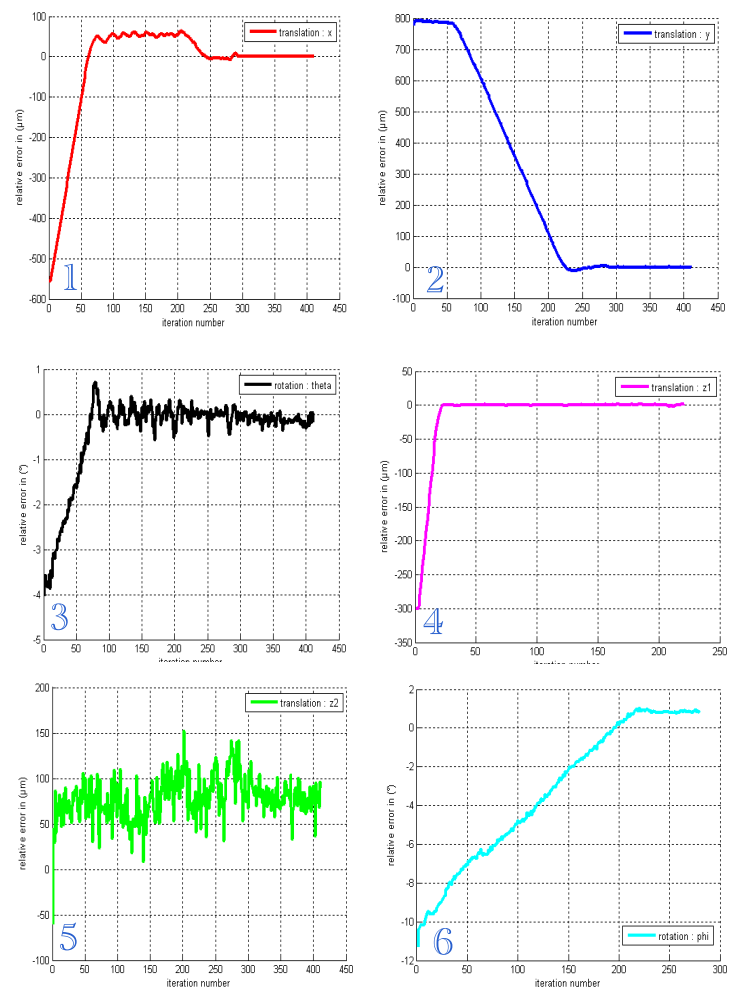

Fig. 8. Image 1 to 6 plot the error between the current and the desired position $x, y, \theta, z_{1}$ (in task\#2), $z_{2}$ (in task\#3) and $\phi$ respectively, versus number of iterations

concentration and dexterity order to avoid the damage of either the system and the parts. The proposed approach performs the same assembly 15 times faster compared to 
the human mode.

That ratio between the time required the human operator and robotic system becomes increasingly important when the human stress increases. This factor creates fatigue in the human would damage the products.

Table 2 presents some assembly examples. In each assembly process, the time spent to achieve the three tasks (task\#1, task\#2 and task\#3) is computed. The final assembly cycle time is presented in the fourth column. The fastest is about 34 seconds, the slowest 47.7 seconds and the mean time for the all tests is about 41 seconds which is an exceptional value for that kind of task.

\begin{tabular}{|c|c|c|c|}
\hline $\mathrm{N}^{\circ}$ & task\#1 (sec) & task\#2+task\#3 (sec) & Total $(\mathrm{sec})$ \\
\hline Assembly 1 & 25.9 & 13.9 & 39.8 \\
\hline Assembly 2 & 29.7 & 11.7 & 41.4 \\
\hline Assembly 3 & 22.0 & 12.0 & 34.0 \\
\hline Assembly 4 & 28.3 & 11.5 & 39.8 \\
\hline Assembly 5 & 35.7 & 11.7 & 47.4 \\
\hline Assembly 6 & 31.3 & 12.0 & 43.3 \\
\hline Assembly 7 & 28.8 & 11.8 & 40.6 \\
\hline Assembly 8 & 29.4 & 11.9 & 41.3 \\
\hline Assembly 9 & 27.8 & 11.9 & 39.7 \\
\hline Assembly 10 & 29.4 & 11.7 & 41.1 \\
\hline Mean & $\mathbf{2 8 . 8}$ & $\mathbf{1 2 . 0}$ & $\mathbf{4 0 . 8}$ \\
\hline
\end{tabular}

TABLE II

SOME ASSEMBLY CYCLE TIME IN SECONDS

\section{Vi. Conclusion and Future Perspectives}

The problem of the robotic microassembly of 3D devices was studied in this paper. Despite the important scientific contributions in the domain, there is still much effort to get the famous flexible, modular, versatile, repeatable and precise process. One solution to that problem is the development of vision based control, particularly 3D visual control. Therefore, this paper addressed the automation of assembly from a conceptual viewpoint to practical solution compatible with industrial constraints.

The task addressed is the assembly of two $400 \mu \mathrm{m} \times 400$ $\mu \mathrm{m} \times 100 \mu \mathrm{m}$ parts by their respective $100 \mu \mathrm{m} \times 100 \mu \mathrm{m}$ $\times 100 \mu \mathrm{m}$ notches with a mechanical play of $3 \mu \mathrm{m}$. The setup mainly comprises:

- a $x y \theta$ and $z \phi$ robotic system,

- a two finger gripping system,

- a videomicroscope.

The process is broken down into three tasks, sequentially achieved: the positioning of the second part, the insertion of the latter in the former. The parts are tracked in realtime by means of an algorithm based on their CAD model. Despite the high level of accuracy and low level of quality, the occlusions of the target, the algorithm gives robust and accurate 3D poses. The 3D measurements delivered by the tracking method are used to implemented accurate 3D control of the distributed robotic system. The considered task was achieved with a good accuracy 15 times faster than a human operator.

The future work will concern the reduction of the above time in order to make it more compatible with industrial requirement. The construction of complex structures will also be investigated.

\section{AKNOWLEDGEMENTS}

This work is partially conducted with financial support from the project Hybrid Ultra Precision Manufacturing Process Based on Positional and Self assembly for Complex Micro-Products (HYDROMEL NMP2-CT-2006026622) funded by the European Commission.

\section{REFERENCES}

[1] F. Chaumette and S. Hutchinson, Visual servo control, part 1: Basic approaches, IEEE Robotics and Automation Magazine 13 (2006), no. 4, 82-90.

[2] L. Chen, W. Rong Sun, L. and, and X. Bian, Hybrid control of vision and force for mems assembly system, IEEE Int. Conf. on Robotics and Biometrics (Shenyang, China), August 2004.

[3] A.I. Comport, E. Marchand, M. Pressigout, and F. Chaumette, Realtime markerless tracking for augmented reality: the virtual visual servoing framework, IEEE Trans. on Visualization and Computer Graphics 12 (2006), no. 4, 615-628.

[4] A.N. Das, P. Zhang, W.H. Lee, D. Popa, and H. Stephanou, Multiscale, deterministic micro-nano assembly system for construction pf on-wafer microrobots, IEEE ICRA'07 (Roma, Italy), April 2007.

[5] N. Dechev, L. Ren, W. Liu, W.L. Cleghorn, and J.K. Mills, Development of a 6 degree of freedom robotic micromanipulation for use in 3d mems microassembly, IEEE ICRA'06 (Orlando, Fl.), May 2006.

[6] R. Devanathan, S. Wenting, S. Chai, and A. Shacklock, Multi view and multi scale image based visual servo for micromanipulation, Studies in Computational Intelligence 8 (2006), 105-133.

[7] T. Drummond and R. Cipolla, Real-time visual tracking of complex structures, IEEE PAMI 24 (2002), no. 7, 932-946.

[8] John T. Feddema and Ronald W. Simon, Visual servoing and caddriven microassembly, IEEE Robotics and Automation Magazine Vol. 5 (4) (1998), 18-24.

[9] R. Haralick, H. Joo, C. Lee, X. Zhuang, V Vaidya, and M. Kim, Pose estimation from corresponding point data, IEEE Trans on Systems, Man and Cybernetics 19 (1989), no. 6, 1426-1445.

[10] P.-J. Huber, Robust statistics, Wiler, New York, 1981.

[11] D.G. Lowe, Fitting parameterized three-dimensional models to images, IEEE PAMI 13 (1991), no. 5, 441-450.

[12] E. Malis, F. Chaumette, and S. Boudet, 2 1/2 D visual servoing, IEEE Trans. on Robotics and Automation 15 (1999), no. 2, 238-250.

[13] E. Marchand, F. Chaumette, F. Spindler, and M. Perrier, Controlling an uninstrumented manipulator by visual servoing, The Int. Journal of Robotics Research, IJRR 21 (2002), no. 7, 635-648.

[14] P. Martinet and J. Gallice, Position based visual servoing using a nonlinear approach, IEEE/RSJ IROS'99 (Kyongju, South Korea), 1999, pp. 531-536.

[15] Y. Mezouar and P.K. Allen, Visual servoed micropositioning for protein manipulation tasks, IEEE/RSJ Int. Conf. on Intelligent Robots and System, IROS'02. 2 (2002), 1766-1771.

[16] B.J. Nelson, S. Ralis, Y. Zhou, and B. Vikramaditya, Force and vision feedback for robotic manipulation of the microworld, Experimental Robotics - VI-LNCIS 250 (1999), 433-442.

[17] B. Tamadazte, S. Dembélé, G. Fortier, and N. Le Fort-Piat, Automatic micromanipulation using multiscale visual servoing, IEEE CASE (Washington, USA), August 2005.

[18] W. Wilson, C. Hulls, and G. Bell, Relative end-effector control using cartesian position-based visual servoing, IEEE Trans. on Robotics and Automation 12 (1996), no. 5, 684-696.

[19] G. Yang, J. Gaines, and B. Nelson, A surpervisory wafer-level 3d microassembly system for hybrid mems fabrication, J. of Intelligent and Robotic Systems 37 (2003), 43-68.

[20] G. Yang and B. Nelson, Integration of microscopic vision and microforce feedback for microassembly, Int. Workshop on Microfactories (Mineapolis, Minnesota), September 2002.

[21] K. Yesin and B. Nelson, A cad-model based tracking system for visually guided microassembly, Robotica 23 (2005), 409-418. 